\title{
Mineral bone disorder in chronic kidney disease: head-to-head comparison of the 5/6 nephrectomy and adenine models
}

\author{
Guaraciaba O Ferrari ${ }^{1}$, Juliana C Ferreira ${ }^{1}$, Raquel T Cavallari ${ }^{1}$, Katia R Neves ${ }^{1}$, Luciene M dos Reis ${ }^{1}$, \\ Wagner V Dominguez ${ }^{1}$, Elizabeth C Oliveira ${ }^{1}$, Fabiana G Graciolli ${ }^{1}$, Jutta Passlick-Deetjen ${ }^{2}$, Vanda Jorgetti ${ }^{1}$ \\ and Rosa MA Moysés ${ }^{1 *}$
}

\begin{abstract}
Background: Experimental models are important to the understanding of the pathophysiology of, as well as the effects of therapy on, certain diseases. In the case of chronic kidney disease-mineral bone disorder, there are currently two models that are used in evaluating the disease: 5/6 nephrectomy ( $N x)$ and adenine-induced renal failure (AIRF). However, the two models have never been compared in studies using animals maintained under similar conditions. Therefore, we compared these two models, focusing on the biochemical, bone histomorphometry, and vascular calcification aspects.
\end{abstract}

Methods: Wistar rats, initially fed identical diets, were divided into two groups: those undergoing 5/6 Nx (5/6Nx group) and those that were switched to an adenine-enriched diet (AIRF group). After 9 weeks, animals were sacrificed, and we conducted biochemical and bone histomorphometry analyses, as well as assessing vascular calcification.

Results: At sacrifice, the mean body weight was higher in the 5/6Nx group than in the AIRF group, as was the mean blood pressure. No differences were seen regarding serum phosphate, ionized calcium, intact parathyroid hormone (PTH), or fibroblast growth factor 23 (FGF23). However, creatinine clearance was lower and fractional excretion of phosphate (FeP) was higher in the AIRF group rats, which also had a more severe form of high-turnover bone disease. Vascular calcification, as evaluated through von Kossa staining, was not observed in any of the animals.

Conclusions: Overt vascular calcification was not seen in either model as applied in this study. Under similar conditions of diet and housing, the AIRF model produces a more severe form of bone disease than does 5/6 Nx. This should be taken into account when the choice is made between these models for use in preclinical studies.

Keywords: Bone disease, Chronic kidney disease, Hyperparathyroidism, Phosphate binder, Vascular calcification

\section{Background}

Chronic kidney disease (CKD) is a worldwide health problem, commonly associated with high morbidity and mortality. One common and challenging complication is CKD-mineral bone disorder (CKD-MBD), defined by abnormalities in mineral and hormone metabolism, as well as bone histological changes, with or without soft tissue calcification [1]. However, many pathophysiological aspects of CKD-MBD are still not completely understood.

\footnotetext{
* Correspondence: rosa.moyses@uol.com.br

${ }^{1}$ Nephrology Department, Universidade de São Paulo, Rua Iperoig, 690, apto 121, São Paulo, Brazil

Full list of author information is available at the end of the article
}

As a result, new therapeutic strategies, although needed, are scarce. In addition, clinical trials that could improve our understanding of CKD-MBD and the potential benefits or hazards of therapeutic interventions are extremely difficult and costly to conduct. In this regard, animal models could be a strategic option, providing the opportunity to examine the basic aspects of this disease, and its treatment.

Until recently, 5/6 nephrectomy (Nx), first described at the beginning of the 20th century [2], was the most common animal model used in preclinical studies evaluating CKD-MBD. However, the 5/6Nx model has several drawbacks: it requires surgical skill; it has a high mortality 
rate; and pronounced MBD takes several weeks to develop [3]. During the last few years, a model originally suggested in the 1980s [4], based on the nephrotoxicity provoked by adenine, has been also employed in this setting. In that model, CKD is induced by administration of adenine in diet or solution, thereby precluding the need for surgery. Authors who have employed the adenine model to evaluate CKD-MBD have reported that it provokes a more severe form of the disease, with impressive bone alterations, as well as significant VC $[5,6]$. Nevertheless, it is difficult to compare the results obtained with these two models, because the dietary protocol employed has not been standardized (neither in terms of the amount and duration of adenine administration, nor in terms of the dietary concentrations of calcium, phosphate, and protein), as well as because the observation period and therapeutic strategies have varied across studies. Therefore, the exact differences between the $5 / 6 \mathrm{Nx}$ and adenine models, as well as which would be the best model to evaluate a specific aspect of bone disease, remain unclear. To answer these questions, we decided to compare both models under identical conditions, in terms of animal species, diet, and duration of CKD.

\section{Methods}

\section{Ethics statement}

All experimental procedures were approved by and conducted in accordance with the guidelines of the Standing Committee on Animal Research of the Universidade de São Paulo School of Medicine (Protocol no. 0447/08).

\section{Experimental protocol}

Thirty five male Wistar rats, weighing 300-350 g, were obtained from our local breeding colony. They were housed in individual cages in a temperature- and humidity-controlled environment $\left(25^{\circ} \mathrm{C}\right.$ at $25 \%$ humidity), on a 12/12-h light/dark cycle, and fed a standard powder diet containing $1.17 \%$ calcium, $0.93 \%$ phosphate, $19.3 \%$ protein, and $4.2 \mathrm{IU} / \mathrm{g}$ vitamin $\mathrm{D}_{3}$ (LabDiet $^{\oplus}$ 5C3Y; PMI Nutrition International, St. Louis, MO, USA), with ad libitum access to water. After 1 week of acclimatization, rats were allocated to be submitted to $5 / 6 \mathrm{Nx}$, which consisted of removal of the right kidney and infarction of approximately $2 / 3$ of the left kidney, or to start an adenine-enriched diet (LabDiet ${ }^{\odot} 5 \mathrm{~K} 52$ plus $0.75 \%$ and 0.50\% adenine; PMI Nutrition International). A normal renal function Control group was also allocated. The Control and the $5 / 6 \mathrm{Nx}$ groups rats were fed the standard diet throughout the study. For the AIRF group, the adenine concentration was $0.75 \%$ in the first two weeks and $0.50 \%$ in the following two weeks. Thereafter, the AIRF group rats were fed the standard diet [7]. A pair-feeding protocol was used. On a weekly basis, the rats were weighed and blood pressure was measured by tail cuff plethysmography. At 9 weeks after the surgical procedure and the start of adenine administration, rats were anesthetized and sacrificed by exsanguination via aortic puncture.

We injected each rat intraperitoneally with $25 \mathrm{mg} / \mathrm{kg}$ oxytetracycline (Terramycin ${ }^{\circledR}$; Pfizer Animal Health, New York, NY, USA), a fluorescent marker of bone, on days $51,52,58$ and 59 of the protocol, for dynamic evaluation of bone histomorphometry. We also collected 24-h urine samples, 1 day before the sacrifice, in order to quantify urine output and perform biochemical analysis.

Serum samples were collected at the time of sacrifice by aortic puncture and stored at $-20^{\circ}$ for subsequent biochemical evaluation. The heart was excised, and the left ventricle was dissected for weighing and determination of the left ventricle weight normalized to body weight $(\mathrm{LVW} / \mathrm{BW})$. Thoracic and abdominal aortas were removed in order to evaluate $\mathrm{VC}$, by von Kossa staining and calcium content quantification, respectively. Femurs were removed for bone histomorphometry.

\section{Biochemical analysis}

In serum samples, we measured creatinine and phosphate by colorimetric assay (Labtest, Lagoa Santa, Brazil); ionized calcium was measured with an ion-selective analyzer (AVL-9140; AVL Scientific Corporation, Roswell, GA, USA); the PTH level was determined by enzymelinked immunosorbent assay (ELISA kit; Immutopics, San Clemente, CA, USA), as was the level of FGF23 (ELISA kit; Kainos Laboratories, Tokyo, Japan); calcitriol was quantified by radioimmunoassay using a pool of samples. In urine samples, we quantified creatinine, phosphate, and calcium by colorimetric assay (Cobas Amplicor; Roche Diagnostics, Indianapolis, IN, USA).

\section{Bone histomorphometry}

The left femur of each rat was removed, dissected free of soft tissue, immersed in 70\% ethanol, and processed as previously described [8]. Using a Jung $\mathrm{K}$ microtome (Reichert Jung, Heidelberg, West Germany), we cut distal femurs into sections of $5 \mu \mathrm{m}$ and $10 \mu \mathrm{m}$ thickness. The $5-\mu \mathrm{m}$ sections were stained with $0.1 \%$ toluidine blue, $\mathrm{pH} 6.4$, and we examined at least two nonconsecutive sections per sample.

Structural, static, and dynamic parameters of bone formation and resorption were measured at the distal metaphysis (magnification, $\times 250$ ), at $195 \mu \mathrm{m}$ from the epiphyseal growth plate, in a total of 30 fields, using a semi-automatic image analyzer (Osteomeasure; Osteometrics, Atlanta, GA, USA). Structural parameters included trabecular thickness (in $\mu \mathrm{m}$ ), trabecular separation (in $\mu \mathrm{m}$ ), and trabecular number (in trabeculae/mm). The indices of static formation included the proportions of trabecular bone volume and osteoid volume to total bone 
volume (both in \%); osteoid thickness (in $\mu \mathrm{m}$ ); and osteoid/osteoblast surfaces (both in \% of bone surface). The indices of static resorption included eroded surface and osteoclast surface (both in \% of bone surface). The mineral apposition rate, determined by measuring the distance between the two oxytetracycline labels and dividing it by the time elapsed between the two oxytetracycline administrations, is expressed in micrometers per day. Mineralization lag time is expressed in days. Determination of the proportion of double oxytetracyclinelabeled surface to total trabecular surface (in \%) and of the bone formation rate completed the dynamic evaluation. Results are also described according to the turnovermineralization-volume (TMV) classification [9]. Histomorphometric indices were reported using nomenclature recommended by the American Society of Bone and Mineral Research [10].

\section{Vascular calcification}

Cross-sections of the thoracic aorta were embedded in paraffin, sectioned at $4 \mu \mathrm{m}$, and stained with von Kossa. For the analysis of calcium concentration, the abdominal aorta was solubilized in $0.6 \mathrm{~N} \mathrm{HCl}(40 \mu \mathrm{L} \mathrm{HCl} / g$ tissue $)$ for $48 \mathrm{~h}$, after which it was dried and weighed. The sample was then centrifuged, and the calcium content was measured in the supernatant (Calcium Reagent Set; Pointe Scientific, Canton, MI, USA).

\section{Statistical analysis}

Results are presented as mean \pm SD or median (min-max). Comparisons among groups were made using Anova. Graph Pad Prism version 3.03 (GraphPad Software, Inc., San Diego, CA, USA) were used. Values with $P<0.05$ were considered as statistically significant.

\section{Results}

Study sample

The final study sample comprised 30 rats: 10 in the $5 / 6 \mathrm{Nx}$ group; 10 in AIRF group; and, 10 in Control group. Although 15 rats were submitted to $5 / 6 \mathrm{Nx}$, three died (on post-procedure days 3,7 , and 50 , respectively) and two others were excluded from the final analysis because of the severity of renal failure (serum creatinine levels of 6.7 and $3.5 \mathrm{mg} / \mathrm{dl}$, respectively). There were no deaths in the AIRF group. As shown in Table 1, despite the use of a pair-feeding protocol, the mean body weight at sacrifice was lower in the AIRF group. Mean urine output was significantly higher in the AIRF group, whereas mean blood pressure was higher in the $5 / 6 \mathrm{Nx}$ group. No differences were seen regarding the LVW/BW.

\section{Biochemical parameters}

Creatinine levels tended to be higher in the AIRF group than in the $5 / 6 \mathrm{Nx}$ group, whereas a significant difference
Table 1 Characteristics of the rats evaluated ${ }^{1}$

\begin{tabular}{lccc}
\hline Variable & $\begin{array}{c}\text { Control } \\
(\mathbf{n}=\mathbf{1 0})\end{array}$ & $\begin{array}{c}\text { AIRF } \\
(\mathbf{n}=\mathbf{1 0})\end{array}$ & $\begin{array}{c}\mathbf{5 / 6 N \mathbf { N }} \\
(\mathbf{n}=\mathbf{1 0})\end{array}$ \\
\hline Initial body weight $(\mathrm{g})$ & $324.4 \pm 37.4$ & $307.1 \pm 30.8$ & $307.9 \pm 19.9$ \\
Final body weight (g) & $366.1 \pm 18.7$ & $312.0 \pm 17.2^{\mathrm{a}}$ & $385.4 \pm 59.8^{\mathrm{a}, \mathrm{b}}$ \\
Food intake (g/day) & $17.5 \pm 1.4$ & $16.8 \pm 1.9$ & $18.9 \pm 0.7^{\mathrm{b}}$ \\
Urine output (ml) & $11.4 \pm 5.4$ & $53.7 \pm 11.2^{\mathrm{a}}$ & $22.7 \pm 6.7^{\mathrm{a}, \mathrm{b}}$ \\
TCP-BP (mmHg) & $119.7 \pm 7.3$ & $154.4 \pm 20.4^{\mathrm{a}}$ & $220.0 \pm 19.5^{\mathrm{a}, \mathrm{b}}$ \\
LWW/100 g BW & $0.18 \pm 0.01$ & $0.21 \pm 0.02^{\mathrm{a}}$ & $0.21 \pm 0.05$ \\
\hline
\end{tabular}

${ }^{1}$ Data expressed as mean \pm SD.

TCP-BP, Blood pressure, as measured by tail cuff plethysmography.

Initial, Before Nx5/6 and adenine diet protocol.

Final, At the sacrifice.

${ }^{a} p<0.05$ vs. Control; ${ }^{b} p<0.05$ vs. AIRF.

regarding creatinine clearance was seen between uremic groups (Table 2). The two groups were comparable in terms ionized calcium, phosphate, PTH, and FGF23. However, despite the similar serum phosphate levels, FeP was higher in the AIRF group. In addition, calcitriol levels were significantly lower in the $5 / 6 \mathrm{Nx}$ group.

\section{Bone histomorphometry}

Bone histomorphometry revealed that the bone formation rate was significantly higher in the AIRF group rats than in the $5 / 6 \mathrm{Nx}$ group rats. Other parameters of bone formation, such as osteoid and osteoblast surfaces, as well as resorption and osteoclast surfaces, were also significantly higher in the AIRF group. The mineralization lag time was higher in AIRF group. We also observed no significant differences between the two groups in terms of trabecular bone volume. However, bone microarchitecture was altered in the AIRF group animals, as evidenced by a lower trabecular number and greater trabecular separation. Fibrosis was observed in only one AIRF rat (Table 3).

Table 2 Biochemical data at the sacrifice

\begin{tabular}{lccc}
\hline Variable & Control $(\mathbf{n}=\mathbf{1 0})$ & AIRF $(\mathbf{n}=\mathbf{1 0})$ & $\mathbf{5 / 6 N x}(\mathbf{n}=\mathbf{1 0})$ \\
\hline Serum Cr (mg/dl) & $0.6 \pm 0.1$ & $1.6 \pm 0.3^{\mathrm{a}}$ & $1.3 \pm 0.6^{\mathrm{a}}$ \\
Creat Clear (ml/min) & $2.55 \pm 0.74$ & $0.67 \pm 0.25^{\mathrm{a}}$ & $1.1 \pm 0.46^{\mathrm{a}, \mathrm{b}}$ \\
Serum iCa (mmol/L) & $1.2 \pm 0.1$ & $1.1 \pm 0.1$ & $1.1 \pm 0.1$ \\
uCa (mg/dl) & $14(8-17)$ & $6(4-8)$ & $11(5-50)$ \\
Serum P (mg/dl) & $5.6 \pm 0.9$ & $7.4 \pm 0.7^{\mathrm{a}}$ & $6.7 \pm 1.4$ \\
FeP $(\%)$ & $9.1 \pm 4.8$ & $21.2 \pm 11.0^{\mathrm{a}}$ & $7.6 \pm 4.6^{\mathrm{b}}$ \\
iPTH (pg/ml) & $108(47-315)$ & $681(163-1,604)^{\mathrm{a}}$ & $572(198-4,199)^{\mathrm{a}}$ \\
FGF23 (pg/ml) & $157(107-310)$ & $784(418-14.476)^{\mathrm{a}}$ & $893(649-2.609)^{\mathrm{a}}$ \\
Calcitriol $(\mathrm{pg} / \mathrm{ml})^{*}$ & $69.2 \pm 17.3$ & $88.0 \pm 16.6$ & $35.1 \pm 20.0^{\mathrm{b}}$ \\
\hline
\end{tabular}

Data expressed as mean \pm SD or median (min-max).

Creat Clear, Creatinine clearance; Serum iCa, Serum ionized calcium; uCa, Urinary calcium; Serum P, Serum phosphate. *Calcitriol was measured in pooled sera.

$\mathrm{P}<0.05^{\mathrm{a}}$ vs Control; ${ }^{\mathrm{b}}$ vs AIRF. 
Table 3 Bone histomorphometry data

\begin{tabular}{|c|c|c|c|}
\hline Parameter & $\begin{array}{l}\text { Control } \\
(n=10)\end{array}$ & $\begin{array}{c}\text { AIRF } \\
(n=10)\end{array}$ & $\begin{array}{c}5 / 6 \mathrm{Nx} \\
(\mathrm{n}=10)\end{array}$ \\
\hline $\mathrm{BV} / \mathrm{TV}(\%)$ & $25.0 \pm 4.8$ & $31.1 \pm 6.7$ & $30.3 \pm 3.7$ \\
\hline $\mathrm{TbN}(\mathrm{n} / \mathrm{mm})$ & $3.7 \pm 0.6$ & $3.3 \pm 0.7$ & $4.7 \pm 0.5^{a, b}$ \\
\hline $\mathrm{Tb} . \mathrm{SP}(\mu \mathrm{m})$ & $208.8 \pm 48.2$ & $223.6 \pm 95.6$ & $147.6 \pm 26.5^{b}$ \\
\hline Tb.Th $(\mu \mathrm{m})$ & $67.1 \pm 5.5$ & $93.4 \pm 8.8^{\mathrm{a}}$ & $63.8 \pm 4.0^{b}$ \\
\hline ObS/BS (\%) & $5.4 \pm 3.0$ & $25.3 \pm 6.2^{a}$ & $5.8 \pm 2.3^{b}$ \\
\hline OV/BV (\%) & $0.45(0.14-0.56)$ & $1.46(0.54-3.78)^{a}$ & $0.28(0.20-0.77)^{b}$ \\
\hline O.Th $(\mu \mathrm{m})$ & $1.7 \pm 0.4$ & $2.6 \pm 1.3$ & $1.5 \pm 0.2^{b}$ \\
\hline OS/BS (\%) & $1.6(1.2-2.1)$ & $30.3(18.8-40.5)^{\mathrm{a}}$ & $6.1(5.1-13.9)^{b}$ \\
\hline OcS/BS (\%) & $1.9 \pm 1.1$ & $7.4 \pm 2.8^{\mathrm{a}}$ & $3.2 \pm 1.5^{b}$ \\
\hline ES/BS (\%) & $9.1 \pm 1.9$ & $24.5 \pm 6.3^{a}$ & $12.3 \pm 5.1^{b}$ \\
\hline MS/BS (\%) & $6.0(3.7-9.1)$ & $10.3(7.1-14.3)$ & $2.4(0.8-5.3)^{a, b}$ \\
\hline $\operatorname{MAR}(\mu \mathrm{m} /$ day $)$ & $0.8 \pm 0.4$ & $1.0 \pm 0.7$ & $0.8 \pm 0.2^{b}$ \\
\hline $\operatorname{BFR}\left(\mu \mathrm{m}^{3} / \mu \mathrm{m}^{2}\right.$ per day $)$ & $0.03(0.01-0.05)$ & $0.14(0.01-0.22)$ & $0.02(0.01-0.03)^{\mathrm{b}}$ \\
\hline $\operatorname{MLT}(d)$ & $3.6(1.6-8.1)$ & $8.4(1.4-136.0)^{\mathrm{a}}$ & $4.6(3.4-14.8)^{b}$ \\
\hline
\end{tabular}

Data expressed as mean \pm SD or median (min-max).

$\mathrm{BV} / \mathrm{TV}$, Bone volume/trabecular volume; TbN, Trabecular number; Tb.SP,

Trabecular separation; TbTh, Trabecular thickness; Obs/BS, Osteoblast surface/ bone surface; OV/BV, Osteoid volume/bone volume; O.Th, Osteoid thickness; OS/BS, Osteoid surface/bone surface; OcS/BS, Osteoclast surface/bone surface; $\mathrm{ES} / \mathrm{BS}$, Eroded surface/bone surface; $\mathrm{MS} / \mathrm{BS}$, Mineralization surface/bone surface; MAR, Mineral apposition rate; BFR, Bone formation rate; MLT, Mineralization lag time.

${ }^{a} \mathrm{p}<0.05$ vs. Control; ${ }^{\mathrm{b} v s .}$ AIRF.

\section{Vascular calcification}

Von Kossa staining was negative in both groups. However, calcium content in the abdominal aorta was significantly greater in the $5 / 6 \mathrm{Nx}$ group (Table 4 ).

\section{Discussion}

To our knowledge, this was the first study to compare, under similar conditions, the most widely used animal models of CKD-MBD. We showed that, in rats fed identical diets and observed for the same length of time, renal dysfunction and bone disease are more severe when the AIRF model is employed.

Initially described in 1932, 5/6Nx (ligation of the renal artery branches, electrocauterization of the renal cortex or removal of the left kidney poles and right kidney nephrectomy) reduces the effective mass of the kidneys [11]. The effectiveness of this model is dependent upon the anatomy of individual animals and the experience of the researcher, leading to different levels of CKD [12].

Table 4 Vascular calcification

\begin{tabular}{lccc}
\hline Parameter & Control & AIRF & 5/6Nx \\
\hline AAC $(\mu \mathrm{mol} / \mathrm{mg})$ & $9.6(8.6-10.6)$ & $7.0(4.7-11.4)$ & $15.1(13.3-17.9)^{\mathrm{a}, \mathrm{b}}$ \\
\hline
\end{tabular}

Data expressed as median (min-max).

AAC, Abdominal aortic calcium

${ }^{a} \mathrm{p}<0.05$ vs. Control; ${ }^{b}$ vs. AIRF.
According to classical descriptions, animals submitted to $5 / 6 \mathrm{Nx}$ develop hyperphosphatemia, reduced calcitriol levels, and hyperparathyroidism, as well as VC and highturnover bone disease [3,13-15]. VC is typically seen at approximately 12 weeks after the surgical procedure unless the animals are fed with a high-phosphate diet or calcitriol is administered to them [16]. Of the 15 rats initially included in our $5 / 6 \mathrm{Nx}$ group, three died. This could be attributed to the surgical procedure itself or to greater renal disease severity. Given that two additional animals were excluded from the final analysis because they had extremely high serum creatinine levels, it is clear that, when the $5 / 6 \mathrm{Nx}$ model is employed, such losses must be taken into account and a higher number of rats should be included in the original sample. Considering the potential expenses involved, this could be viewed as a disadvantage of the $5 / 6 \mathrm{Nx}$ model in comparison with the AIRF model.

The adenine model of nephrotoxicity was first described in 1982, when Yokozawa et al. observed that 2.8-dihydroxyadenine, a metabolite of adenine, precipitated as crystals in renal tubules, causing rats fed an adenine-rich diet to develop uremia [4]. Since then, adenine-enriched diets or solutions of adenine, in various concentrations, have been used by many authors for varying periods of time and for different purposes. In 2003, Katsumata et al. used this model, feeding rats a $0.75 \%$ adenine diet for 3 weeks in order to study the effects of sevelamer [5]. In 2006, Tamagaki et al. analyzed the bone and metabolic characteristics of AIRF in rats after 2, 4, and 6 weeks on a $0.75 \%$ adenine diet and showed that VC develops only after 4 weeks, whereas incipient bone disease was seen at 2 weeks, leading to a severe skeletal impairment after 6 weeks [6]. In that same year, Nagano et al. published the results of an experiment involving a different dose of adenine $(0.75 \%$ for 2 weeks followed by $0.50 \%$ for 2 weeks) and showed that the rats developed renal failure, as well as hyperphosphatemia, secondary hyperparathyroidism, and reduced calcitriol levels [7]. Subsequent studies involved different adenine concentrations and periods of administration $[5-7,12,14,17,18]$. So, it seems clear that the degree of renal dysfunction, as well as the extent of bone disease and VC, is totally dependent on the amount of adenine administered and the duration of administration.

One important element in the construction of an experimental model of CKD is the amount of calcium and phosphate administered in the diet, as well as the source of phosphate (grain or casein). The bioavailability of phosphate is greater for diets based on casein (cow-milk protein) than for those based on grain [19]. In addition, the phosphate load should be quantified by measuring the FeP, given that serum phosphate might not increase if parathyroid function is preserved since PTH increases 
renal phosphate excretion. Therefore, in the present study, we designed an experiment in which we tested the $5 / 6 \mathrm{Nx}$ and AIRF under identical conditions in terms of the calcium, phosphate (from grain), and protein content of the diet.

We found that the AIRF group rats had lower weight, despite the pair-feeding protocol, as has been reported previously [6]. This has been attributed to the poor palatability of adenine, as well as to the uremia and polyuria caused by tubular disease, which in addition to the ingestion of a minimal amount of water, could lead to dehydration and weight loss [20]. This finding should be interpreted as a limitation of AIRF.

The 5/6Nx model induces a progressive glomerular sclerosis, whereas the adenine model promotes intratubular crystal precipitation and interstitial nephritis. Based on this, we would expect to find proteinuria, hypertension and cardiac hypertrophy in the first model, whereas acidosis and more severe bone lesions would be seen in the second model. In our experiment, the LVW/BW was similar between the two groups, despite the higher blood pressure observed in the $5 / 6 \mathrm{Nx}$ group. This was probably because the AIRF group rats showed greater uremia, which is also a risk factor for ventricular hypertrophy (in fact, we found a positive correlation between serum creatinine and LVW/BW; $\mathrm{R}=0.69, \mathrm{p}<0.05$ ). Therefore, the $5 / 6 \mathrm{Nx}$ model is indicated for studies evaluating ventricular hypertrophy associated with hypertension, whereas the adenine model would be more appropriate for the study of other variables that could lead to ventricular hypertrophy.

We found the two groups to be comparable in terms of serum and urinary calcium, as well as serum phosphate. However, $\mathrm{FeP}$ was found to be significantly higher in the AIRF group, which could be explained by the increased bone turnover or by tubulointerstitial nephropathy, with direct tubular damage leading to phosphaturia. In favor of the latter theory, we found no statistically significant correlation between FeP and the two major phosphaturic hormones, PTH and FGF23, nor between $\mathrm{FeP}$ and bone resorption parameters (data not shown). However, a direct effect of adenine on the tubular excretion of phosphate has never been shown. Our data also show that there were no significant differences between the two models in terms of PTH or FGF23. Various authors have reported an extreme increase in PTH levels in the adenine model. However, some [6] administered adenine for a longer period time, whereas others measured PTH using assays that were different from ours, impeding comparisons across studies. In addition, as previously mentioned, none of the previous studies compared the adenine model to $5 / 6 \mathrm{Nx}$ model in a single experiment. Surprisingly, we found higher calcitriol levels in AIRF than in Nx group. However, calcitriol levels in AIRF were not different from Control group, results that are similar to those were described by Damment et. al, who found no significant differences in calcitriol levels between normal renal function and chronic renal failure, adenine-treated rats [17]. The reasons for this unexpected finding could be the combination of non-significant higher PTH levels and lower FGF-23 in adenine-treated rats or a technical issue with calcitriol measurements, which are usually done in pools of sera, due to the significant amount of serum that is necessary for this procedure or even an increased calcitriol synthesis in the interstitial renal granulomas that are usually seen around adenine crystals [21].

The bone effects of $5 / 6 \mathrm{Nx}$ and AIRF are distinct, confirming previous findings that the natural history of CKD-MBD could be also dependent on the model that was chosen to perform the experiments [22,23]. In our study, we found a high-turnover bone disease in AIRF. Notable among our histomorphometric findings are the bone formation rate and the proportion of osteoid surface, which were 5 times higher in the AIRF group rats than in the $5 / 6 \mathrm{Nx}$ group rats. We also found a positive correlation between serum creatinine and bone formation rate $(\mathrm{R}=0.59 ; \mathrm{p}<0.05)$, showing that worse renal function leads to higher bone turnover. Nevertheless, osteoblasts were apparently unable to increase bone matrix deposition to the same degree, probably because this process is not only cell-mediated but also physicochemical, as AIRF caused greater phosphate loss in urine, probably impairing mineralization. In addition, accumulating evidence suggests that extracellular nucleotides, signaling through P2 purinoceptors, play an important role in modulating bone cell function. Adenosine triphosphate and other nucleotides can stimulate the formation and resorptive activity of osteoclasts, as well as inhibiting bone mineralization by osteoblasts [24]. Because adenosine is derived from adenine, a purine base, we cannot rule out the hypothesis that high doses of adenine lead to an increase in adenosine, which might have direct effects on the function of osteoblasts and osteoclasts. However, we did not observe any crystal in bone marrow in our samples. Another possibility is that the lower calcitriol levels described in some adenine animal studies could be responsible for the observed increase in osteoid parameters; however, in the present study, calcitriol levels were significantly higher in the AIRF group than in the $5 / 6 \mathrm{Nx}$ group. Although metabolic acidosis could also influence bone findings in the adenine model, we did not measure whole blood $\mathrm{pH}$, which could be viewed as a limitation of our study. However, a recent study employing the adenine model, in which serum creatinine at the end of the study was similar to that described in our experiment, showed levels of $\mathrm{pH}$ and $\mathrm{CO}_{2}$ that could not explain the magnitude of the bone findings, 
making it less probable that metabolic acidosis is a relevant factor [17]. Nevertheless, despite the fact that the cause of the increased amount of osteoid observed in the adenine model remains unknown, this finding should be kept in mind when choosing a model to evaluate the bone effects of certain drugs, such as phosphate binders. The administration of these drugs in the adenine model of CKD could increase osteoid parameters, as previously shown by Neven et al. [18].

We found it surprising that the von Kossa staining was negative in both groups and that the calcium concentration in the abdominal aorta was higher in the $5 / 6 \mathrm{Nx}$ group. This would seem to be in disagreement with the current concept that the AIRF frequently leads to VC. However, our animals received less adenine than did those described in the study conducted by Tamagaki et al. [6], which treated animals for 6 weeks with $0.75 \%$ adenine and observed massive VC. Therefore, it is likely that our protocol of adenine administration was insufficient to provoke the expected VC. In relation to $5 / 6 \mathrm{Nx}$, other studies employing this model have shown that, in nephrectomized rats, $\mathrm{VC}$ is not detected through von Kossa staining in normal conditions [3]. Therefore, it is understandable that the von Kossa staining was negative in the $5 / 6 \mathrm{Nx}$ group rats, since our study had a duration time of 9 weeks. Another question is why the $5 / 6 \mathrm{Nx}$ group rats presented higher calcium concentration in the abdominal aorta than did the AIRF group rats. One hypothesis is that the duration of CKD could be different in the two models. Another possibility is that the high blood pressure in $5 / 6 \mathrm{Nx}$ model could lead to a higher calcium deposition. Therefore, if one plans to evaluate massive $\mathrm{VC}$, experimental animals must be fed higher concentrated adenine-diet for a longer period of time or must be observed after several months in the 5/6Nx model [25].

Our study presents several limitations: we did not measure biochemical variables or performed histomorphometric analysis at intermediate time points; we did not evaluate different concentrations of adenine in the diet; we could not separate the effects of PTH, FGF-23 or calcitriol, or kidney function in each of these models. In addition, the duration of our experiment mimics approximately $4-5$ years in human disease and it is known that CKD-MBD has an insidious course of many years. However, we were able to demonstrate for the first time in a head-to-head comparison, that animals present different forms of CKD depending on the model that is chosen. In face of our results, 5/6 Nx model should be employed to study the effects of intervention on blood pressure, on kidney disease progression, on serum levels of PTH, FGF-23 and calcitriol or on mild bone lesions of secondary hyperparathyroidism. It should also be used in studies with longer observation period. On the other hand, AIRF model should be used to evaluate ventricular hypertrophy not related to hypertension or volume overload. The direct effect of adenine crystals on renal tubules and interstitium impairs its use on studies of effects of CKD-MBD therapy on CKD progression. Long term studies are also difficult with this model because there is a progressive undernutrition and severe renal disease, unless lower concentrations of adenine are employed.

\section{Conclusion}

In summary, our study shows the peculiarities of the adenine and $5 / 6 \mathrm{Nx}$ models in terms of the CKD-MBD induced. Its main strength is that, for the first time, $5 / 6 \mathrm{Nx}$ and adenine models were compared under similar conditions of housing, feeding, and observation period. Using the same experimental protocol for both models, we found that the adenine model provokes massive phosphaturia and a more severe form of bone disease, although we observed no significant differences between the two models in terms of VC. Nevertheless, the differences identified should be taken into account when a specific model is selected in order to evaluate the effects of CKD-MBD therapies.

\section{Competing interests}

The authors declare that they have no competing interests.

\section{Authors' contributions}

GOF: performed the experiments, analyzed the data, wrote the manuscript; JCF: performed the experiments; RTC: performed the experiments; KRN: conceived and designed the experiments, performed the experiments; LMR: performed the experiments; WD: performed the experiments; ECO: performed the experiments; FGG: performed the experiments; JPD: conceived and designed the experiments, VJ: conceived and designed the experiments; RMAM: conceived and designed the experiments, performed the experiments, analyzed the data, wrote the manuscript. All authors read and approved the final manuscript.

\section{Acknowledgements}

The authors are grateful for the assistance provided by Jefferson D. Boyles in the translation and editing of the text, as well as for the technical assistance provided by Rosimeire A. Bezerra Costa, Grasiela Pedreira Barlette, and Cássia Macedo Pucci.

\section{Funding}

This study was supported by Fresenius Medical Care Deutschland GmbH, Germany and Fapesp (grant 2008/53147-0). RMAM is supported by CNPQ, Conselho Nacional de Desenvolvimento Científico e Tecnológico (grant 303325/2010-0). It was presented in part at the ASN Kidney Week 2011.

\section{Author details}

Nephrology Department, Universidade de São Paulo, Rua Iperoig, 690, apto 121, São Paulo, Brazil. 'Division of Nephrology, University of Dusseldorf, Dusseldorf, Germany.

Received: 10 December 2013 Accepted: 30 April 2014

Published: 3 May 2014

\section{References}

1. Kidney Disease: Improving global outcomes (KDIGO) CKD-MBD work group KDIGO clinical practice guideline for the diagnosis, evaluation, prevention, and treatment of chronic kidney disease-mineral and bone disorder (CKD-MBD). Kidney Int Supp/ 2009, 113:S1-S130.

2. Chauntin A, Ferris EB: Experimental renal insufficiency produced by partial nephrectomy. Arch Intern Med 1932, 49:767-787. 
3. Shobeiri N, Adams MA, Holden RM: Vascular calcification in animal models of CKD: a review. Am J Nephrol 2010, 31:471-481.

4. Yokozawa T, Oura T, Okada T: Metabolic effects of dietary purine in rats. J Nutr Sci Vitaminol 1982, 28:519-526.

5. Katsumata K, Kusano K, Hirata M, Tsunemi K, Nagano N, Burke SK, Fukushima N: Sevelamer hydrochloride prevents ectopic calcification and renal osteodystrophy in chronic renal failure rats. Kidney Int 2003, 64:441-450.

6. Tamagaki K, Yuan Q, Ohkawa H, Imazeki I, Moriguchi Y, Imai N, Sasaki S, Takeda K, Fukagawa M: Severe Hyperparathyroidism with bone abnormalities and metastatic calcifications in rats with adenine-induced uraemia. Nephrol Dial Transplant 2006, 21:651-659.

7. Nagano N, Myata S, Abe M, Kobayashi N, Wakita S, Yamashita T, Wada M: Effect of manipulating serum phosphorus with phosphate binder on circulating PTH and FGF23 in renal failure rats. Kidney Int 2006, 69:531-537.

8. Gouveia CH, Jorgetti V, Bianco AC: Effects of thyroid hormone administration and estrogen deficiency on bone mass of female rats. J Bone Miner Res 1997 12:2098-2107.

9. Moe S, Drueke T, Cunningham J, Goodman W, Martin K, Olgaard K, Ott S, Sprague S, Lameire N, Eknoyan G: Definition, evaluation, and classification of renal osteodystrophy: a position statement from Kidney Disease: Improving Global Outcomes (KDIGO). Kidney Int 2006, 69:1945-1953.

10. Dempster DW, Compston JE, Drezner MK, Glorieux FH, Kanis JA, Maluche H, Meunier PJ, Ott SM, Recker RR, Parfitt AM: Standardized symbols, and units for bone histomorphometry: a 2012 update of the report of the ASBMR Histomorphometry Nomenclature Committee. J Bone Miner Res 2013, 28:2-17.

11. Gagnon RF, Duguid WP: A reproducible model for chronic renal failure in the mouse. Urol Res 1983, 11:11-14.

12. Terai K, Mizukami K, Okada M: Comparison of chronic renal failure rats and modification of the preparation protocol as a hyperphosphataemia model. Nephrology 2008, 13:139-146.

13. Oste L, Behets G, Dams G, Bervoets AR, Marynissen RL, Geryl H, van Hoof VO, de Broe ME, D'Haese PC: Role of dietary phosphorus and degree of uremia in the development of renal bone disease in rats. Ren Fail 2007, 29:1-12.

14. Nagano N, Myiata S, Obana S, Kobayashi N, Abe M, Fukushima N, Wada M: Sevelamer hydrochloride, a calcium free phosphate binder, inhibits parathyroid cell proliferation in partially nephrectomized rats. Nephrol Dial Transplant 2003, 18(3):81-85.

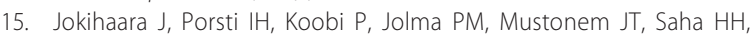
Sievanen H, Kannus P, Iwaniec UT, Turner RT, Jarvinen TL: Treatment of experimental renal osteodystrophy with pamidronate. Kidney Int 2008, 74:319-327.

16. Graciolli FG, Neves KR, dos Reis LM, Graciolli RG, Noronha IL, Moysés RM, Jorgetti V: Phosphorus overload and PTH induce aortic expression of Runx2 in experimental uraemia. Nephrol Dial Transplant 2009, 24(5):1416-1421.

17. Damment S, Secker R, Shen V, Lorenzo V, Rodriguez M: Long-term treatment with lanthanum carbonate reduces mineral and bone abnormalities in rats with chronic renal failure. Nephrol Dial Transplant 2011, 26:1803-1812.

18. Neven E, Dams G, Postnov A, Postnov A, Chen B, de Clerck N, de Bro ME, D'Haese PC, Persy V: Adequate phosphate binding with lanthanum carbonate attenuates arterial calcification in chronic renal failure rats. Nephrol Diet Transplant 2009, 14:1-10.

19. Moe S, Chen N, Seifert MF, Sinders RM, Duan D, Chen X, Liang Y, Radcliff JS, White KE, Gattone VH 2nd: A rat model of chronic kidney disease-mineral bone disorder. Kidney Int 2009, 75:176-184.

20. Ali BH, Ziada A, Al Husseni I, Beegam S, Nemmar A: Motor and behavioral changes in rats with adenine-induced chronic renal failure: influence of acacia gum treatment. Exp Biol Med 2011, 236(1):107-112.

21. Okabe C, Borges RL, de Almeida DC, Fanelli C, Barlette GP, Machado FG, Arias SC, Malheiros DM, Camara NO, Zatz R, Fujihara CK: NF-kB activation mediates crystal translocation and interstitial inflammation in adenine overload nephropathy. Am J Physiol Renal Physiol 2013, 15(305(2)):F155-F163.

22. Sabbagh Y, Graciolli FG, O'Brien S, Tang W, dos Reis LM, Ryan S, Phillips L, Boulanger J, Song W, Bracken C, Liu S, Ledbetter S, Dechow P, Canziani ME, Carvalho AB, Jorgetti V, Moyses RM, Schiavi SC: Repression of osteocyte
Wnt/ $\beta$-catenin signaling is an early event in the progression of renal osteodystrophy. J Bone Miner Res 2012, 27(8):1757-1772.

23. Stubbs JR, He N, Idiculla A, Gilihan R, Liu S, David V, Hong Y, Quarles LD: Longitudinal evaluation of FGF23 changes and mineral metabolism abnormalities in a mouse model of chronic kidney disease. J Bone Miner Res 2012, 27(1):38-46.

24. Orris I, Burnstock G, Arnett T: Purinergic signaling and bone remodeling. Curr Opin Pharmacol 2010, 10(3):322-330.

25. Cozzolino M, Staniforth M, Liapis H, Finch J, Burke SK, Dusso AS, Slatopolsky E: Sevelamer Hydrochloride attenuates kidney and cardiovascular calcifications in long-term experimental uremia. Kidney Int 2003, 64:1653-1661.

doi:10.1186/1471-2369-15-69

Cite this article as: Ferrari et al:: Mineral bone disorder in chronic kidney disease: head-to-head comparison of the 5/6 nephrectomy and adenine models. BMC Nephrology 2014 15:69.

\section{Submit your next manuscript to BioMed Central and take full advantage of:}

- Convenient online submission

- Thorough peer review

- No space constraints or color figure charges

- Immediate publication on acceptance

- Inclusion in PubMed, CAS, Scopus and Google Scholar

- Research which is freely available for redistribution 